
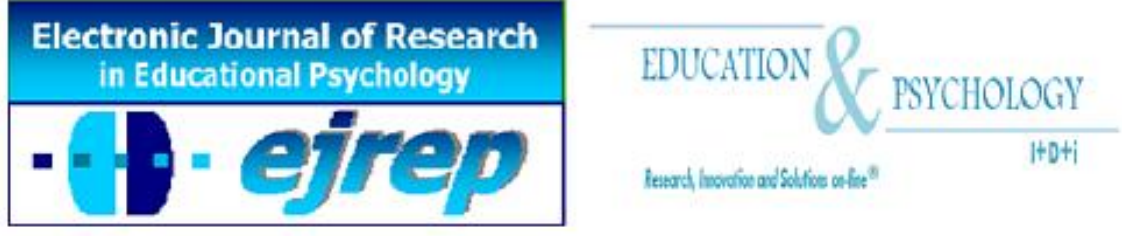

\title{
Percepciones y Expectativas del Profesorado Palestino ante la Inclusión Educativa en el Distrito de Belén
}

\author{
${ }^{1}$ ABU-HERAN, N., ${ }^{2}$ ABUKHAYRAN, A., \\ ${ }^{3}$ DOMINGO, J. y ${ }^{4}$ PÉREZ-GARCÍA, M.P.
}

\author{
${ }^{1}$ Candidata a Doctora, Universidad de Granada \\ ${ }^{2}$ Profesor Titular, Facultad de Educacion, Al-Quds University, Jerusalem \\ ${ }^{3}$ Catedrático de Universidad, Facultad CC. Education, Universidad Granada \\ ${ }^{4}$ Profesora Titular Universidad, Facultad CC. Educacion, Universidad Granada
}

\section{Palestina y España}

Correspondencia: Jesús Domingo Segovia. Facultad de Ciencias de la Educación, Campus de Cartuja, s/n, 18071 Granada (Spain). E-mail: jdomingo@ugr.es

(c) Education \& Psychology I+D+i and Editorial EOS (Spain) 


\section{Resumen}

Introducción. El estudio indaga sobre la realidad de la educación inclusiva en Palestina en opinión de su profesorado. Presenta del marco legislativo y social de la educación palestina, y lo pone en contraste con el conocimiento actual en el tema de la educación inclusiva. Desde ahí se extraen una serie de elementos clave para la inclusión. El profesorado es determinante para posibilitar o no la inclusión en el aula; por eso interesan sus percepciones y expectativas ante el reto y la realidad de la inclusión educativa.

Método. Recoge la opinión de una muestra representativa del profesorado que trabaja en clases ordinarias con chicos con necesidades educativas especiales del Sector de Belén. Utiliza una metodología descriptiva, inferencial y correlacional, mediante un cuestionario. Se señalan los resultados más interesantes en relación a las variables de estudio en las que se concentran las dificultades: estudiantes, profesorado, padres, centro, currículum, actividad extraescolar y formación para la vida, gestión del aula, dirección/gestión, metodología didáctica, conciencia y actitud.

Resultados. Como en el cuestionario se pregunta sobre el estado real y deseable de la cuestión, se identifican los escenarios posibles en los que se podría avanzar desde una perspectiva posibilista y de sentido común, así como los elementos que contarán con mayor resistencia entre el profesorado. Los resultados informan que el profesor palestino es consciente de la realidad y de las dificultades para afrontar el reto de la educación inclusiva. Hay una fuerte crítica con la realidad y, al mismo tiempo, la conciencia de que se deben tomar algunas medidas para que sea posible.

Discusión y conclusión. La inclusión educativa está aún en sus primeros pasos en Palestina, pero existe un marco de esperanza. El profesorado es crítico con su realidad y muestra vías de acción para avanzar hacia la inclusión. Ofrecer y asumir estas medidas es esencial para un sistema que se encuentra dando sus primeros pasos hacia la inclusión y que también tiene el afán de conquistar y construir su futuro en esta línea de acción.

Palabras Clave: Integración escolar; educación inclusiva; actitud del profesorado; necesidades educativas especiales; retos educativos. 


\section{Perceptions and Expectations of Palestinian Teachers towards Inclusive Education in Bethlehem District}

\section{Abstract}

Introduction. The study investigates about the reality of inclusive education in Palestine according to the opinion of teachers. It is presented in the legislative and social framework of education in Palestine and compares it with current knowledge on inclusive education, from which a series of essential elements for inclusive education can be derived. Teachers are a key to achieving inclusion of students with special educational needs in ordinary classroom; so it is critical to take into account their perceptions of the challenges and realities of inclusion and their expectations.

Method. The summary gathers the opinions of a representative sample of the teachers including students with special educational needs in ordinary classrooms in the Bethlehem sector. A descriptive methodology is used, and results attained by inference and correlations from respondents of a questionnaire. The most important results are taken from the study variables in the areas in which difficulties occur such as: the students with disabilities, teachers, parents, administration, work environment (school), curriculum, classroom management, extracurricular activities, methods and teaching aids, awareness and attitudes.

Results. As in the questionnaire, questions on the real and desirable state of inclusive education are posed and from the responses possible scenarios are identified in which there can be progress from a positive and common sense perspective. The results inform us that the Palestinian teacher is aware of the reality and difficulties in facing the challenge of inclusive education. There is strong criticism and at the same time awareness of some measures that should be taken to make it possible.

Discussion and conclusion. The educational inclusion is still in its infancy in Palestine, but there is a hope framework. The teaching is critical to their reality shows and ways of action to move towards inclusion. To give and to take these measures is is essential to a System that finds itself taking its first steps towards inclusion and it also has the eagerness of conquering and building the future.

Keywords: school integration, inclusive education, teachers' attitudes, special educational needs, educational challenges. 


\section{Introducción}

El estudio gira en torno a las opiniones del profesorado palestino acerca de la presencia cada vez más generalizada de todo tipo de alumnado en sus clases y el progreso de la inclusión educativa. Pretende con ello conocer la percepción del profesorado en torno a los retos y dificultades que encuentran la integración y la inclusión educativa de chicos y chicas con discapacidad en Palestina. De modo que se pueda mostrar una panorámica de la situación en torno a diez dimensiones de análisis y con dos enfoques diferentes: percepción de la realidad del estado de la cuestión, y expectativas sobre lo que es la situación óptima.

Como alertan Bruns \& Mogharreban (2007), en torno a la inclusión son claras las diferencias entre creencias y prácticas. A partir de los grados de sintonía y discrepancia de estos resultados, se puede establecer un mapa de posibilidad de integración y hoja de ruta para la mejora de la educación inclusiva en Palestina. Estos resultados pretenden ser un elemento más a debate para, de una parte, generar conciencia y estado de opinión sobre el tema, como primer paso para cambiar las perspectivas del profesorado y las actitudes de todas las partes interesadas, y, de otra, buscar vías alternativas justas que mejoren la situación y ofrezcan oportunidades reales de inclusión en Palestina.

\section{Educación Inclusiva en Palestina}

Desde el último cuarto del siglo pasado se viene defendiendo la presencia y la atención eficiente de todo el alumnado en el sistema educativo. Sin violentar dicha premisa, aún es motivo de continuos debate acerca de la integración y de su eficacia, así como sus posibles efectos positivos (Kugelmass, 2004; Barton, 2005; Miles, 2005). A tal efecto, la Declaración de Salamanca (UNESCO, 1994) supuso un antes y un después en el tema, con una presencia cada vez más evidente en declaraciones internacionales (ONU, 2006) y en impacto en los sistemas educativos, apareciendo propuestas, instrumentos e indicadores que facilitaran dicho proceso (Ainscow, 1999; Booth \& Ainscow, 2002; Villa \& Thousand, 2005; Ainscow, Booth, \& Dyson, 2006). En las últimas décadas y todos los países se ha reconocido cada vez más oficialmente el principio de la educación inclusiva como vía pertinente de escolarización de los niños con discapacidades en los centros ordinarios; ya que es "el medio más eficaz de construir una sociedad integradora y lograr la educación para todos" (Sentenac et al., 2013; UNESCO, 1994a). 
La integración escolar de alumnado con discapacidad ganó un importante reconocimiento internacional, cuando Naciones Unidas (ONU) promovió la idea de la 'Educación para Todos' en la Conferencia Mundial en Tailandia en 1990 (Khochen \& Radford, 2012; Kuyini \& Desai, 2007; UNESCO, 2012a); siendo actualmente la inclusión de personas con discapacidad en la educación y la sociedad en general una tendencia mundial (Rafael et al., 2013).

Actualmente la comunidad internacional considera la inclusión educativa una cuestión ética y de justicia, y aunque está aún lejos de conseguirse, debe promocionarse más allá de cualquier otra consideración (Barton, 2005; Echeita et al., 2009; Echeita \& Ainscow, 2011). La educación inclusiva se fundamente en el derecho de todos los niños y jóvenes a una educación de calidad, con independencia de sus diferencias, posiciones o capacidades. Del mismo modo resalta los valores educativos de la equidad, la diversidad y la justicia social (Moran, 2007). Por todo ello, la inclusión educativa ocupa un lugar preponderante en el ámbito de la literatura pedagógica actual, incluso con un potente elenco de revistas de impacto que se ocupan de mostrar los avances más destacados de la investigación en el ámbito social y educativo en esta temática.

La comunidad árabe, en general y la palestina en particular, no han quedado al margen del tema y han seguido sus propios procesos. De este modo, el Ministerio de Educación y Educación Superior (MEES) de Palestina ha adoptado la definición de la UNESCO para la educación inclusiva. Y establece que la educación debe ofrecer igualdad de oportunidades educativas para todos los estudiantes, y encontrar una forma adecuada para atender las diversas necesidades que puedan aparecer en la educación escolar. También adoptó la Declaración de Salamanca. En los artículos 2 y 3 se regula la aceptación en las escuelas de todos los estudiantes con discapacidades, y en 55 se regula la enseñanza de las niñas con discapacidad. Dice buscar el "Garantizar los derechos de los discapacitados a tener igualdad de oportunidades para asistir a las instituciones educativas y las universidades, en el marco de los planes de estudios adoptado en estas instituciones" (Palestinian Legislative Council, Law on Rights of Disabled Palestinians, 1999, p. 2; Palestinian Ministry of Education, 2008).

La educación inclusiva se inició en Palestina con una fase piloto entre 1997-1999 con el apoyo de Diakonia / NAD, la UNESCO y Save the Children-Suecia. El programa se amplió para incluir a todas las escuelas del país durante el período 2006-2007, donde se crearon comités de amigos de los discapacitados, y un maestro fue nominado responsable de la educa- 
ción inclusiva (maestro de tiempo completo con un programa de enseñanza completo, como una persona contacto entre el asesor del ministerio y los estudiantes). Los aspectos del programa más criticados fueron que no existían criterios objetivos para el proceso de integración, la falta de profesorado especializado, la carga de trabajo del profesor responsable y la falta de fondos (Karlsson, 2004; Al-Sabbah et al, 2008).

Por ello, la UNESCO, dentro de la ayuda internacional para el desarrollo educativo (UNESCO, 2012b), creó la Estrategia Nacional de Apoyo a la Educación en Palestina (UNESS). El desarrollo del programa UNESS requiere desempeñar un papel mucho más estratégico en el apoyo a las necesidades y prioridades nacionales de educación y en el fortalecimiento de la asociación con otros organismos para el desarrollo, en conformidad con los objetivos de desarrollo internacionales.

Al igual que en otros países, la realidad de la situación está lejos de ser la deseada y todavía tiene un largo camino por recorrer, y es en las primeras etapas de desarrollo (Climent, 2009) dando -no sin dificultades- unos primeros pasos de escolarización, normalización e integración escolar. Palestina, aún con una norma relativamente actualizada y con avances evidentes en algunos programas, propuestas y escuelas, no puede garantizar de momento una educación inclusiva y el éxito para todos.

Al reflexionar sobre este punto, queda claro que hay una necesidad actual para crear una mayor conciencia sobre el derecho a una educación inclusiva y de su necesidad para los estudiantes con discapacidad en Palestina. Asimismo es necesario conocer las percepciones de los profesores sobre esta realidad e iniciativa.

\section{Percepciones del profesorado hacia la Educación Inclusiva}

La educación de estudiantes con dificultades de aprendizaje o discapacidades ha cambiado drásticamente en las últimas dos décadas y varios países han llevado a cabo esfuerzos para poner en práctica políticas que fomenten la integración y, más recientemente, la inclusión de este alumnado en los entornos ordinarios. Aunque la inclusiva educativa ha cobrado impulso en los últimos años, un elemento clave para su implementación exitosa es la opinión de quien tiene la responsabilidad de su puesta en acción. Las creencias y actitudes de los docentes son fundamentales para garantizar el éxito de las prácticas inclusivas. La aceptación y el compromiso de los mismos con la inclusión afecta a su logro (Avramidis \& Norwich, 2002). 
El profesorado influye fuertemente en la implementación de la educación inclusiva en cualquier país (Kim, 2011). Algunos estudios realizados sobre las actitudes del profesorado y sus opiniones sobre la discapacidad, los estudiantes con discapacidad y la inclusión o la integración, indica claramente que el éxito de cualquier política de inclusión es altamente dependiente de estas actitudes y creencias (Ojok \& Wormnaes, 2012; Thaver \& Lim , 2012).

Las actitudes hacia este alumnado son un aspecto clave para su integración en aulas ordinarias o en otras actividades sociales. Cuando los estudiantes tienen una actitud positiva hacia los compañeros con discapacidad puede facilitar su inclusión, mientras que una actitud negativa puede impedirla (De Laat et al., 2013; Vignes et al., 2009). Del mismo modo, las actitudes indiferentes o incluso negativas por parte del profesorado ordinario tiene también obvias repercusiones perjudiciales en el alumnado que necesita medidas de apoyo, y dan lugar a sentimientos de alienación, angustia psicosocial, vulnerabilidad y un profundo sentimiento de ser "menor", debido a su discapacidad (Thaver \& Lim, 2012).

El profesorado de las escuelas ordinarias tiene la responsabilidad de satisfacer las necesidades y los intereses de todo su alumnado, incluido los que tienen diferentes tipos de discapacidades o riesgo de desventaja. Las actitudes y la disposición del profesorado de primaria para enseñar al alumnado con discapacidad intelectual en las escuelas regulares es uno de los factores críticos para la implementación exitosa de la educación inclusiva (Ojok \& Wormnaes, 2012).

\section{Objetivos}

El objetivo de este estudio es doble: de una parte, comprobar si el profesorado palestino es consciente de la realidad y las dificultades para hacer frente al desafío de la educación inclusiva en Palestina y si quiere cambiarla; y, de otra, identificar las dimensiones principales sobre las que se asienta su opinión y sobre las que habría que incidir para su mejora.

\section{Método}

\section{Participantes}

El estudio se desarrolló en el año académico 2012/2013 en Belén. La población es todo el profesorado palestino (1-12 grados) que enseñan en aulas regulares que incluyen estudiantes 
con discapacidades en sus escuelas de Belén y que trabajan para el gobierno, las UNRWA o escuelas privadas. La población incluye 1008 maestros y 1732 maestras. Se selecciona esta población por varios motivos: fue el primer distrito donde se implementó el programa de integración escolar y puede considerarse como representativa de la realidad palestina. Reúne todos los requisitos y variaciones de escuelas y es considerada como una zona política, social y educativa media, tanto en términos de población como de número de escuelas (Lempinen \& Repo, 2002). La muestra del estudio se compone de 340 profesores. Esta muestra fue obtenida por un sistema de selección aleatoria estratificada, y se calculó utilizando la Web http://www.surveysystem.com/sscalc.htm, que es un calculador de tamaño muestral, con un margen de error de 0,05 .

\section{Instrumentos}

Para responder a los objetivos de investigación se utiliza un cuestionario elaborado específicamente con tal fin: Las dificultades de la integración de los estudiantes palestinos con discapacidad a las aulas regulares. Estudio de Bethelehem (realidad y el deseo). Se construye a partir de la revisión teórica y de otros instrumentos utilizados para analizar la realidad de la inclusión en Palestina. El instrumento para la recolección de datos consta de dos secciones. La primera recoge las características demográficas del profesorado palestino. La segunda, incluye los retos y dificultades de la integración de los estudiantes palestinos con discapacidad en las aulas regulares en torno 107 ítems repartidos entre diez dimensiones: Retos y dificultades relativos al alumnado, al profesorado, a las familias, a la Administración Educativa, al ambiente de trabajo en la escuela, los programas escolares, a la gestión de la clase, las actividades extracurriculares, los métodos y medios de enseñanza utilizados, y la toma de conciencia y cambio de actitud.

Se usa una escala líker de cuatro opciones $(4=$ Muy de acuerdo, $3=$ De acuerdo, $2=$ En desacuerdo y 1 = Muy en desacuerdo). Se usa tanto para que opinen sobre el estado actual o la realidad de la inclusión educativa en Palestina, como para expresar sus expectativas sobre lo que consideraría más adecuado y posible. El cuestionario reúne los parámetros de calidad exigibles al propósito en cuanto a fiabilidad y validez. Se cuidó la validez de contenido a través del juicio de 24 expertos profesionales en ejercicio y especializados en el tema de diversas instituciones universitarias y de la Administración Educativa. La validez de constructo se aseguró mediante una potente revisión teórica y el análisis factorial: los componentes esenciales resultantes superaban el $50 \%$ de la varianza y coincidían con las dimensiones objeto de 
estudio. Y, por último, en cuanto a validez predictiva o de criterio, se aplicó la prueba de fiabilidad de Alfa de Cronbach, tanto global, como por dimensiones y escalas, obteniendo un alto índice de fiabilidad: Alfa de Cronbach, global del cuestionario es de .965 . En la escala que describe la realidad, es de $.950 \mathrm{y}$, por dimensiones oscila entre $, 949 \mathrm{y}, 951$. Mientras que en la escala que describe las expectativas o lo que consideran que debiera ser, es de .980 y con una oscilación por dimensiones entre .979 y .980 .

\section{Procedimiento}

Se contactó con el Ministerio de Educacion Palestino y se le presentó tanto el proyecto de investigación como el instrumento de recogida de información. Se integraron en él sus aportes y sugerencias. Las autoridades dieron acceso a las escuelas del sector de Belen e instaron a los directores escolares a que presenten y pidan a su profesorado que cumplimente los cuestionarios. Se recogieronn y analizaron los datos. Y, por último, se realizarón los análisis correlaciones e inferenciales que se presentan a continuación.

\section{El análisis estadístico}

Para recoger las evidencias precisas para este análisis, se ha procedido a hacer tres tipos de análisis. De un lado, los descriptivos básicos para la obtención de las medidas de tendencia central. Calculando las medias aritméticas y las medianas que marcan la opinión del profesorado por dimensiones y factores. A partir del análisis factorial por dimensiones se han obtenido las dimensiones esenciales que agrupan y explican la mayor variabilidad del cuestionario.

Como toda esta indagación sobre el estado real y deseable de la cuestión, se obtienen tres panorámicas. En una primera, se describe la realidad de la inclusión palestina. En la segunda, se muestra un escenario de lo que el profesorado cree como deseable. Un tercer análisis, permite identificar otras evidencias de coincidencia y cercanía entre realidad y deseo/posibilidad de cambio. Para ello se analizan las correlaciones bivariadas de los componentes esenciales de cada factor, cruzando realidad y deseo.

Se parte de la premisa de que la necesidad de cambio se puede detectar a partir de la diferencia significativa entre la realidad o lo que se tiene y el deseo. Las diferencias significativas entre realidad y deseo muestran los escenarios posibles en los que se podría avanzar desde una perspectiva posibilista y de sentido común, mientras que el resto de los elementos 
contarán con mayor resistencia entre el profesorado al considerarse más consolidados o no necesarios de cambiar.

\section{Resultados}

El estudio tuvo como objetivo identificar las percepciones y expectativas del profesorado palestino hacia la educación inclusiva en el distrito de Belén. Estos resultados se basan en sus respuestas en el cuestionario. Los resultados se presentan aquí de acuerdo con las preguntas de estudio.

La tabla $n^{\circ}(1)$, muestra los principales resultados del estudio. En ella se describen las principales dimensiones que agrupan las respuestas con mayor variabilidad. Seguidamente se muestran el porcentaje de variabilidad explicada y la opinión media, tanto para el análisis de la realidad, como para la del deseo. Y se completa con una comparativa, que señala tanto la diferencia de medias entre realidad y deseo, como y principalmente el grado de correlación existente entre ambas perspectivas. Todo es descrito con mayor grado de detenimiento en los apartados siguientes de resultados.

Tabla 1. Correlación de los componentes esenciales extraídos de análisis factorial

\begin{tabular}{|c|c|c|c|c|c|c|c|}
\hline \multirow[b]{2}{*}{ 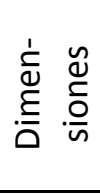 } & \multirow[b]{2}{*}{$\begin{array}{l}\text { Componentes esenciales ex- } \\
\text { traídos de análisis factorial }\end{array}$} & \multicolumn{2}{|c|}{ Realidad } & \multicolumn{2}{|c|}{ Deseo } & \multicolumn{2}{|c|}{ Comparativo } \\
\hline & & $\begin{array}{l}\% \text { Va- } \\
\text { rianza }\end{array}$ & Media & $\begin{array}{l}\% \text { Va- } \\
\text { rianza }\end{array}$ & Media & $\begin{array}{l}\text { Diferen- } \\
\text { cia de } \\
\text { medias }\end{array}$ & $\begin{array}{l}\text { Correla } \\
\text { rela- } \\
\text { ciones } \\
\end{array}$ \\
\hline \multirow{3}{*}{ 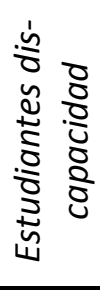 } & $\begin{array}{l}\text { Ante el abandono y el rechazo en } \\
\text { las clases ordinarias }\end{array}$ & 28,554 & 2,6961 & 36,997 & 2,1628 & $-0,533$ &,$- 16^{* *}$ \\
\hline & $\begin{array}{l}\text { Integración personal y social de } \\
\text { los estudiantes }\end{array}$ & 19,021 & 2,797 & 17,34 & 2,879 & 0,0819 & ,027 \\
\hline & $\begin{array}{l}\text { No aceptar estudiantes con dis- } \\
\text { capacidad en las escuelas }\end{array}$ & 10,550 & 2,574 & $*$ & $*$ & $-0,411$ & ,019 \\
\hline \multirow{3}{*}{ 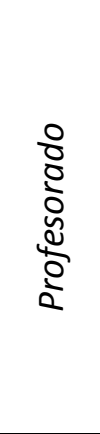 } & $\begin{array}{l}\text { Inadecuada capacitación y apoyo } \\
\text { al profesorado }\end{array}$ & 31,469 & 3,0915 & 41,055 & 2,6196 & $-0,4719$ &,- 093 \\
\hline & $\begin{array}{l}\text { Inadecuada formación y no utili- } \\
\text { zar metodologías para atender a } \\
\text { la diversidad }\end{array}$ & 10,413 & 2,904 & \multirow[b]{2}{*}{10,08} & \multirow[b]{2}{*}{2,935} & 0,0309 &,- 097 \\
\hline & $\begin{array}{l}\text { Aceptación de alumnado con } \\
\text { discapacidad y de los requisi- } \\
\text { tos que ayudan al éxito de la } \\
\text { integración }\end{array}$ & 9,751 & 2,779 & & & 0,1559 &, $23^{* *}$ \\
\hline
\end{tabular}




\begin{tabular}{|c|c|c|c|c|c|c|c|}
\hline \multirow{2}{*}{ 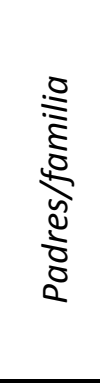 } & $\begin{array}{l}\text { Los padres de estudiantes con } \\
\text { discapacidad carecen de la con- } \\
\text { ciencia necesaria para la integra- } \\
\text { ción de sus hijos }\end{array}$ & 45.625 & 2,6106 & \multirow{2}{*}{55,257} & \multirow{2}{*}{2,5543} & 0,0563 & \multirow{2}{*}{$\begin{array}{l}-, 103 \\
, 002\end{array}$} \\
\hline & $\begin{array}{l}\text { Los padres de estudiantes con } \\
\text { discapacidad no aceptan la } \\
\text { integración de sus hijos en las } \\
\text { escuelas ordinarias }\end{array}$ & 10.413 & 2,962 & & & $-0,4075$ & \\
\hline \multirow{3}{*}{ 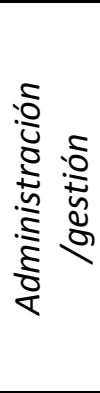 } & $\begin{array}{l}\text { Apoyo insuficiente y falta de ser- } \\
\text { vicios y de claridad en las compe- } \\
\text { tencias }\end{array}$ & 37,738 & 2,9422 & \multirow{3}{*}{59,196} & \multirow{3}{*}{2,4724} & $-0,4698$ &,- 078 \\
\hline & $\begin{array}{l}\text { Los directivos de escuela no } \\
\text { aceptan la integración }\end{array}$ & 8,957 & 2,242 & & & 0,2305 &, $122^{*}$ \\
\hline & $\begin{array}{l}\text { El Sistema Educativo no pro- } \\
\text { porciona las posibilidades ne- } \\
\text { cesarias para la integración }\end{array}$ & 8,480 & 2,869 & & & $-0,397$ & ,038 \\
\hline 岀 $\frac{0}{\mathrm{~S}}$ & $\begin{array}{l}\text { El ambiente escolar no es propi- } \\
\text { cio para el éxito del programa de } \\
\text { integración }\end{array}$ & 47,233 & 3,0373 & 65,959 & 2,4527 & $-0,5846$ &,$- 119^{*}$ \\
\hline \multirow{3}{*}{ 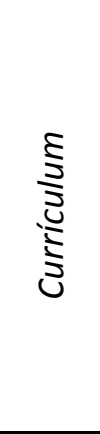 } & $\begin{array}{l}\text { El plan de estudios no contribuye } \\
\text { a promover el proceso de inte- } \\
\text { gración }\end{array}$ & 39,622 & 2,9064 & \multirow{2}{*}{60,751} & \multirow{2}{*}{2,5317} & $-0,3747$ &, $383^{* *}$ \\
\hline & $\begin{array}{l}\text { El plan de estudios no desarro- } \\
\text { lla actitudes positivas hacia los } \\
\text { alumnos con discapacidades }\end{array}$ & 11,634 & 2,505 & & & 0,0272 &, $188^{* *}$ \\
\hline & $\begin{array}{l}\text { El plan de estudios da el tiempo } \\
\text { necesario para el alumnado con } \\
\text { necesidades especiales }\end{array}$ & 8,589 & 2,788 & 8,522 & 2,531 & $-0,256$ & ,080 \\
\hline \multirow{2}{*}{ 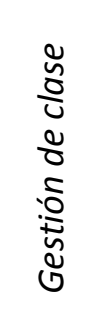 } & $\begin{array}{l}\text { No se utilizan formas correctas } \\
\text { de gestión del aula }\end{array}$ & 38,181 & 2,4993 & 53,944 & 2,4204 & $-0,0789$ &, $208^{* *}$ \\
\hline & $\begin{array}{l}\text { No se utilizan el mismo método } \\
\text { de castigo y recompensa con } \\
\text { todos los estudiantes y tienen } \\
\text { dificultad de tomar en cuenta las } \\
\text { diferencias individuales }\end{array}$ & 13,876 & 2,925 & 11,12 & 2,664 & $-0,261$ & ,081 \\
\hline \multirow{2}{*}{ 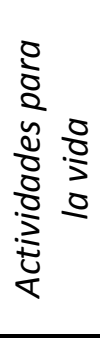 } & $\begin{array}{l}\text { Los estudiantes con discapacida- } \\
\text { des no están involucrados en las } \\
\text { visitas y excursiones, no practi- } \\
\text { can deporte afectiva y activida- } \\
\text { des con otras escuelas } \\
\end{array}$ & 55,684 & 2,7156 & 57,855 & 2,5290 & $-0,1866$ & ,000 \\
\hline & $\begin{array}{l}\text { Formación para la Vida (salidas, } \\
\text { ropa, comida ...) }\end{array}$ & & & 16,965 & 2,825 & 0,1093 & ,040 \\
\hline \multirow{2}{*}{ 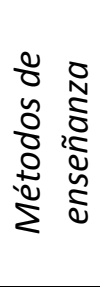 } & $\begin{array}{l}\text { No uso de métodos y medios de } \\
\text { enseñanza para los estudiantes } \\
\text { con discapacidad }\end{array}$ & 43,694 & 2,7037 & 58,249 & 2,4674 & $-0,2363$ &, $376^{* *}$ \\
\hline & $\begin{array}{l}\text { Uso apropiado de recursos y } \\
\text { métodos de enseñanza para es- } \\
\text { tudiantes con discapacidad }\end{array}$ & 9,588 & 2,602 & 9,948 & 2,775 & 0,173 & ,079 \\
\hline
\end{tabular}




\begin{tabular}{|c|c|c|c|c|c|c|c|}
\hline 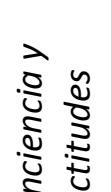 & $\begin{array}{l}\text { La falta de especialistas, normas, } \\
\text { actitudes adecuadas, formación, } \\
\text { el seguimiento y la supervisión } \\
\text { de la integración }\end{array}$ & 42,510 & 2,8738 & 42,510 & 2,4619 & $-0,4119$ & ,016 \\
\hline ర & $\begin{array}{l}\text { Ausencia de actividades y for- } \\
\text { mación sobre integración }\end{array}$ & 8,459 & 2,949 & & & $-0,4866$ &,- 014 \\
\hline
\end{tabular}

Percepciones del profesorado de la realidad de la educación inclusiva

Primero: Retos y dificultades relacionadas con estudiantes con discapacidades. Tres dimensiones principales explican el $58.125 \%$ of the variance of responses en el análisis factorial de la dimensión:

- Frente a la negligencia y rechazo en las escuelas regulares $(28.554 \%$ y mediana $=3)$. De acuerdo a las medianas obtenidas, el profesorado opina que no está de acuerdo con que los estudiantes con discapacidades estén expuestos a la mala conducta de otros estudiantes, golpes o burlas, pero sí identifican que hay dificultad en el resto de los ítems del bloque.

- Integración personal y social de los estudiantes con discapacidades $(19.021 \%$ y mediana $=$ 3). El profesorado está de acuerdo con que la integración y la existencia de programas previos ayuda al desarrollo personal y social de este tipo de alumnado.

- No aceptar estudiantes con discapacidad en las escuelas ordinarias (10.550\% y mediana = 3). Ello denota que el profesorado encuestado está de acuerdo en que su alumnado no aceptan a chicos con discapacidades en su escuela.

Segundo: Retos y dificultades relacionadas con el profesorado. En cuanto a dificultades del profesorado señalan que éstas se agrupan en torno a los siguientes factores:

- Capacitación y apoyo inadecuados para el profesorado $(31.469 \%$ y mediana $=3)$. Reconocen que carecen de formación, medios, recursos, capacidades, etc. para hacer frente a la integración.

- No utilizan los métodos correctos para tratar a los estudiantes con discapacidad $(10,413 \%$ y Mediana = 3).

- Aceptan los programas de integración de los estudiantes con discapacidad y los requisitos que ayudan al éxito de la integración $(9,751 \%$ y mediana $=3)$.

Tertero: Retos y dificultades relacionadas con padres.En lo relativo a los padres, son dos los factores que explican la variabilidad de opinión del profesorado: 
- Los padres de los estudiantes con discapacidad carecen de la conciencia necesaria de la integración de sus hijos en las escuelas ordinarias $(45,625 \%$ y mediana $=3)$.

- Los padres de estudiantes con discapacidades no están aceptando la integración de sus hijos en las escuelas ordinarias $(15,539 \%$ y mediana $=3)$.

Cuarto: Retos y dificultades relacionadas con la administración. Las dificultades percibidas sobre la administración educativa se agrupan en torno a:

- Apoyo inadecuado, falta de servicios de salud y falta de claridad en las responsabilidades y competencias $(37,738 \%$ y Mediana $=3)$.

- Los directores de la escuela no están aceptando la idea de la integración (8.957\%). Al obtener una mediana de 2, significa que no están de acuerdo con esta percepción, lo que denota una fortaleza para la integración: el apoyo de los directores escolares.

- Sistema Educativo no proporciona las posibilidades necesarias para la integración $(8.480 \%$ y mediana $=3)$. Detectan deficiencias significativas en el sistema como para dar un apoyo aceptable a la inclusión en las escuelas palestinas.

Quinto: Retos y dificultades relacionadas con Centro (Escuela). Están de acuerdo (mediana $=3$ ) al opinar que el entorno escolar no es propicio para el éxito del programa de integración. Este componente explica el 47.233 \% de la varianza de respuesta.

Sexto: Retos y dificultades relacionadas con Curriculum. El 59,845\% ode la varianza de las respuestas se explican entre tres componentes esenciales, estando de acuerdo en todos ellos (mediana $=3$ ) en las siguientes opiniones:

- El plan de estudios no contribuye a promover el proceso de integración. (39,622\%).

- No desarrolla actitudes positivas hacia el alumnado con discapacidad (11,634\%).

- No ofrece el tiempo necesario para los estudiantes con necesidades especiales de evaluación $(8,589 \%)$.

Séptimo: Retos y dificultades relacionadas con la gestión del aula. En cuanto a la gestión del aula se observan los siguientes elementos de comprensión:

- No están muy de acuerdo (mediana $=2$ ) con que el profesorado no utiliza las formas correctas de gestión de la clase (39622\%). 
- En cambio sí opinan (mediana = 3) que no utilizan los mismos sistemas de castigo y recompensa con todos los estudiantes y muestran dificultad en tener en cuenta las diferencias individuales $(13,876 \%)$.

Octavo: Retos y dificultades relacionadas con las actividades extracurriculares. La dimension que explica el 55,684\% concluye (mediana $=3$ ) que los estudiantes con discapacidad no están involucrados en las visitas y excursiones, no practican deporte efectivo, y no van a actividades con otras escuelas.

Noveno: Retos y Dificultades relacionadas con metodología didáctica. El profesorado está de acuerdo (mediana $=3$ ) a la hora de enjuiciar los dos factores contradictorios que integran y marcan la mayor variabilidad de respuesta en cuanto a métodos de enseñanza.

- De una parte, afirman que se da un incumplimiento de los métodos y medios de enseñanza para estudiantes con discapacidad $(43,694 \%)$. Opinan que los métodos que utilizan no son motivantes ni apropiados, no fomentan el aprendizaje ni la participación activa, ni reflejan la vida real de este tipo de alumnado.

- Mientras que, seguidamente, afirman que los métodos de enseñanza se utilizan adecuadamente para este alumnado y tienen recursos en las aulas $(9,588 \%)$.

Décimo: Retos y Dificultades relacionadas con conciencia y actitud. El profesorado está de acuerdo con los enunciados (mediana $=3$ ) de los factores que explican el 50,970\% de la varianza de las respuestas de concientización y actitudes:

- La falta de especialistas adecuados, folletos, estudios, seminarios, actividades y programas de radio de la escuela sobre los estudiantes con discapacidad, conocimiento legislación conciencia. $(42,510 \%)$

- Las actividades que faltan y la información sobre la integración $(8,459 \%)$.

El deseo y los retos del profesorado sobre la educación inclusiva

Primero: Estudiantes con discapacidades. El 54,334\% de la varianza de las respuestas se concentra en torno a las siguientes dimensiones: De una parte, el profesorado querría que no hubiese dificultad de integración $(36,997 \%)$; pero consideran que aún habrá dificultades particulares en diferentes aspectos: Ausencia de programas para preparar y organizar al 
alumnado con discapacidad para entrar en las escuelas regulares (programas preescolares). Están de acuerdo (mediana $=3$ ) en la necesidad de ofrecer ayuda a la integración, pues ella aumenta la motivación, el aprendizaje y las relaciones personales de este colectivo de alumnado $(17,337 \%)$.

Segundo: Profesorado. Las dimensiones principales que explican 59,334\% de la varianza de las respuestas son:

- Consideran que aún recibirán un inadecuado servicio de apoyo para el profesorado $(41,055 \%)$, aunque desearían una mejora al respecto, pues baja significativamente en 0,4719 la puntuación media de sus respuestas.

- Se consideran de acuerdo (mediana $=3$ ) con aceptar los programas de integración de estudiantes con discapacidad y con los requisitos que ayudan al éxito de la integración $(10,082 \%)$.

Tertero: Padres. Con una explicación del 55,257\% de la varianza de las respuestas, el profesorado tiene unas bajas expectativas (mediana $=3$ ) sobre el cambio en las familias. De este modo, querrían estar en un futuro próximo en desacuerdo con que los padres de estudiantes con discapacidades necesitan conocer la importancia la integración de sus hijos en las escuelas regulares.

Cuarto: dirección/gestión/Administración. El profesorado querría un adecuado apoyo de los servicios de salud y la claridad en sus facultades $(59.196 \%$ de la varianza de las respuestas), pues no están de acuerdo con la situación actual.

Quinto: Centro (Escuela). Concentrando un 65,959\% de la varianza, el profesorado no querría estar de acuerdo en el futuro (mediana $=2$ ) con que el ambiente escolar no fuese propicio para el éxito del programa de integración.

Sexto: Curriculum. El 69,273\% de la varianza de las respuestas se concentran en las siguientes afirmaciones o dimensiones; aunque con un cierto grado de desacuerdo con las mismas $($ mediana $=2$ ): El plan de estudios no contribuirá a promover el proceso de integración (60,751\%); El plan de estudios no permitirá dar el tiempo necesario para los estudiantes con necesidades especiales $(8,522 \%)$ 
Séptimo: Gestión del aula. Los cambios deseados se concentran en torno a dos dimensiones que explican el 65,062\% de la varianza de las respuestas. Pese a mostrar leves descensos en sus respectivas medias de opinión, desearían un futuro en el que profesorado utilizara medidas correctas de gestión de la clase $(53,944 \%)$. Si bien son conscientes de la dificultad de este cambio y están de acuerdo en que a medio plazo el profesorado deje de utilizar el mismo método de castigo y recompensa con todos los estudiantes y afronte el reto de tener en cuenta las diferencias individuales $(11,118 \%)$.

Octavo: Actividades extracurriculares. Con un $74,820 \%$ de la varizan de respuesta, dos dimensiones toman especial relevancia: Consideran que se debería de ir dejando paulatinamente participar en las visitas y excursiones, practicar deportes, afectos y actividades con otras escuelas a los estudiantes con discapacidad (57,855\%); así como consideran oportuno (mediana $=3$ ) que se les proporcionara formación para la vida (autonomía y rutinas para salidas, vestido, higiene, comida...) $(16,965 \%)$.

Noveno: metodología didáctica. El 68,197\% de la varianza de las respuestas se explica en torno a los siguientes aspectos: De una parte, no desean (mediana $=2$ ) el incumplimiento de los métodos y medios de enseñanza para los estudiantes con discapacidad (58,249\%). Y, de otra, están de acuerdo (mediana= 3) con mejorar los métodos de enseñanza para estudiantes con discapacidades y la presencia y uso adecuado de recursos en el aula $(9,948 \%)$.

Décimo: conciencia y actitud. El 50,970\% de la varianza de respuestas se presenta en dos dimensiones: De un lado, bajar el grado de incumplimiento de los métodos y medios de enseñanza para los estudiantes con discapacidad (42,510\%). Al tiempo que seguir avanzando en utilizar adecuadamente los métodos de enseñanza para estudiantes con discapacidad y aumentar la presencia de recursos $(8,46 \%)$.

\section{Las posibilidades de mejora: hoja de ruta a seguir}

En una primera comparativa se observa que existe una necesidad de cambio con respecto a la inclusión educativa en Palestina a diverso nivel. Esta necesidad de cambio se concentra en las dimensiones donde se observa una correlación altamente significativa (** nivel 0,01). Éstos serían los ámbitos prioritarios de mejora a juicio del profesorado y los que más fácilmente serían admitidos por estos profesionales. En este sentido destacar: 
- Trabajar por un curriculum que vaya abriendo posibilidades del proceso de integración $\left(, 383^{* *}\right)$. En este sentido opinan que se deberían tener elementos curriculares diversificados que permitiesen la creatividad y la adaptación a la diversidad, se definan mejor los niveles adecuados de desempeño de este alumando, se mejoren los indicadores de evaluación, etc.

- Promover la existencia de especialistas, formación del profesorado, el conocimiento de la norma en materia de integración y el uso de los métodos y medios de enseñanza adecuados para los estudiantes con discapacidad $\left(, 376^{* *}\right)$. De manera que se disminuya la influencia negativa de este alumnado en el rendimiento del resto, se superen prejuicios y aumente la satisfacción del profesorado y las familias.

- Aumentar el grado de aceptación de los programas de integración de los estudiantes con discapacidad y el apoyo para el éxito de la integración $\left(, 234^{* *}\right)$. Para lo que proponen mejorar y aumentar la formación (inicial y permanente) recibida para enfrentarse al reto de la inclusión.

- Que el profesorado use formas correctas de gestión de la clase (,208 $\left.{ }^{* *}\right)$. Para lo que proponen promover entre el profesorado el manejo de herramientas para controlar la diversidad de la clase, para la modificación de conducta, o para observar y gestionar el aprendizaje de todos, así como aumentar el grado de comunicación con alumnado y padres, utilizando frases positivas y sin estereotipos...

- Tener en cuenta en el curriculum y en el desarrollo de los procesos de enseñanza y aprendizaje el desarrollo de actitudes positivas hacia la discapacidad $\left(, 188^{* * *}\right)$.

- El profesorado desearía un descenso significativo en las dificultades de integración de este alumnado. Opinan que debe cambiarse la situación de integración en clase, mejorando la atención de estos chicos y colectivos, siendo atendidos como necesitan, pues tienen derecho, y eliminando situaciones de acoso, exclusión o discriminación $\left(-, 163^{* *}\right)$.

Seguidamente, recogiendo otras más sutiles diferencias significativas de opinión (* nivel 0,05) entre la realidad y el deseo, destacar los siguientes resultados:

- Los directores de escuela deben continuar aceptando la idea de la integración educativa y trabajar activamente por su desarrollo $\left(, 122^{*}\right)$.

- El profesorado desea una mejora del ambiente de clase en un sentido mucho más inclusor $\left(-, 119^{*}\right)$, incluso realizando cambios estructurales en la escuela: Considera que debe mejorarse el acceso a las instalaciones y clases, promover la movilidad y la comunicación, 
mejorar y adaptar los recursos y equipamientos, así como promover espacios, tiempos y actividades en los que participen el alumnado con necesidades educativas especiales.

El resto de dimensiones que ha mostrado el análisis factorial del cuestionario, señalan que no tiene correlación significativa, lo que informa que, a juicio del profesorado- son ámbitos relativamente consolidados y en los que -de momento- no es posible el cambio, de manera fácil o sin resistencias significativas.

\section{Discusión y conclusiones}

La inclusión educativa está todavía en sus inicios en Palestina, pero hay un marco de esperanza. Los resultados informan que el profesorado palestino es consciente de su realidad y dificultades para afrontar el reto de la educación inclusiva. Se muestra relativamente crítico con la misma y, al mismo tiempo, es consciente de algunas medidas que debieran acometerse para hacerla posible. Estos deseos, demuestran la posibilidad de tomar medidas esenciales para un sistema que se encuentra dando sus primeros pasos hacia la inclusión y también tiene el afán de conquistar y construir el futuro.

De acuerdo con Kim (2011), Thaver \& Lim (2012) o McDougall et al. (2004), cuando afirman que para hacer posible el cambio es imprescindible contar con el profesorado, tomar en consideración su voz y mirada privilegiada e interesada, como una clave de éxito en el proceso. Ello dotaría de más sentido al cambio propuesto (Fullan, 2007) que, si sólo es un tema administrativo o normativo, difícilmente va a calar en la realidad del aula y la escuela. Incluso si la educación inclusiva es obligatoria por ley, nunca tendrá éxito sin el apoyo entusiasta de sus practicantes. Hay que ser cautos. La obtención de este tipo de apoyo requiere un proceso de cambio de comportamiento y de actitud que no es rápido ni fácil.

El profesorado palestino ofrece una panorámica que a grandes trazos coincide con lo ya mostrado en otros países y estudios anteriores (De Boer, Pijl, \& Minnaert, 2011), por lo que algunas de las medidas exitosas adoptadas como reactivos a tales situaciones, debidamente contextualizadas y estratégicamente implementadas, bien podrán suponer un importante acicate para el Sistema Educativo Palestino. Pero también se deben haber aprendido algunas lecciones por el cambio que tampoco convendría olvidar (Fullan, 2007). 
La investigación internacional señala $-y$ este estudio también lo ha puesto de manifiesto- una compleja mezcla de creencias positivas de los maestros combinadas con temores e insuficiencias percibidas es bastante común en la evolución de la práctica hacia entornos de aprendizaje inclusivos (Shevlin et al, 2012). En este sentido destacar que el profesorado demanda una mejor formación tanto específica como de la profesión en general (Khochen \& Radford, 2012). Opina también que es necesario caminar por el establecimiento de ambientes de aprendizaje inclusivos, e incluso más conscientes de que la diversidad no es sólo una cuestión de capacidades (Shevlin et al, 2012; Slee 2011; Florian et al, 2010). Y, para ello, se debería trabajar en la línea de instaurar dinámicas y valores para que todo el alumnado y la propia idea de inclusión sean bien percibidos por todos (Beyene \& Tizazu, 2010). Para el éxito de la educación inclusiva es importante que profesorado, directivos y otros interesados en la educación deben estar firmemente convencidos de los beneficios que las prácticas inclusivas conllevan a todo el alumnado (Habayeb \& Othman, 2005).

Con todo ello, se puede concluir que la educación inclusiva está en marcha en Palestina. Y, aunque puede encontrarse obviamente en sus fases iniciales (escolarización e integración para la normalización), comienza a existir un primigenio estado de opinión entre el profesorado que alienta a que puede caminar. Lo que requiere el pertinente apoyo y ser conscientes de la envergadura del reto y del volumen de cuestiones que deben ir retocándose: compromiso institucional y de la Administración Educativa, sistema de formación inicial y permanente del profesorado, rediseño del currículum, cambio cultural de las escuelas, estructuras y dinámicas de clase y de escuela, incremento de los niveles de participación e interacción, implicación de las familias y la comunidad, etc.

\section{Referencias}

Ainscow, M., Booth, T., \& Dyson, A. (2006). Improving schools, developing inclusion (Vol. 5). Londres: Routledge.

Ainscow, M. (1999). Understanding the Development of Inclusive Schools (Studies in Inclusive Education Series). London: Routledge Falmer.

Al-Sabbah, S., Khamees, S., Shaeekha, S., Shireen, A., \& Saeed. M. (2008). Obstacles encounter the integration of disabled students in the primary governmental schools in Palestine. Palestine: The Palestinian Ministry of Education. 
Avramidis, E., \& Norwich, B. (2002). Teachers' attitudes towards integration/inclusion: A review of the literature. European Journal of Special Needs Education, 17(2), 129147.

Barton, L. (2005). Special Educational Needs: an alternative look. (A Response to Warnock M. 2005: Special Educational Needs - A New Look). Available from: www.leeds.ac.uk/disability-studies/archiveuk/barton/Warnock.pdf

Beyene, G., \& Tizazu, Y. (2011). Attitudes of Teachers towards Inclusive Education in Ethiopia. Ethiopian Journal of Education and Sciences, 6(1), 89-96.

Booth, T., \& Ainscow, M. (2002). Index for Inclusion. Bristol, UK: Centre for Studies on Incl usive Education.

Bruns, D., \& Mogharreban, C. (2007). The gap between beliefs and practices: Early childhood practitioners' perceptions about inclusion. Journal of Research in Childhood Education, 21(3), 229-241.

Climent, G. (coord.) (2009). La educación inclusiva. De la exclusión a la plena participación de todo el alumnado. Barcelona: Horsori.

De Boer, A., Pijl, S. J., \& Minnaert, A. (2011). Regular primary school teachers' attitudes towards inclusive education: A review of the literature. International Journal of Inclusive Education, 15(3), 331-353.

De Laat, S., Freriksen, E., \& Vervloed, M. P. (2013). Attitudes of children and adolescents toward persons who are deaf, blind, paralyzed or intellectually disabled. Research in developmental disabilities, 34(2), 855-863.

Cohen, L. y Manion, L. (2002). Métodos de Investigación Educativa. Madrid: La Muralla

Echeita, G., \& Ainscow, M. (2011). Inclusive education as a right. Framework and guidelines for action for the development of a pending revolution. Tejuelo, 12, 26-46.

Echeita, G., Simón, C., Verdugo, M.A., Sandoval, M., López, M., Calvo, I., \& González-Gil, F. (2009). Paradojas y dilemas en el proceso de inclusión educativa en España. Revista de Educación, 349, 153-178.

Florian, L., Young, K., Rouse, M. (2010). Preparing teachers for inclusive and diverse educational environments: Studying curricular reform in an initial teacher education course. International Journal of Inclusive Education, 14(7), 709-722.

Fullan, M. (2007). Las fuerzas del cambio con creces. Madrid: Akal.

Habayeb, A. \& Othman, A. (2005). Principals and teachers attitudes towards including the handicapped in regular classes. Nablus, Palestine: Al- Najah National University. 
Karlsson, P. (2004). Towards inclusive education for all in Palestine. A follow-up study of Inclusive Education Project, Ministry of Education Final report. Institute of Public Management: Diakonia/NAD, Reahbilitation Program.

Khochen, M \& Radford, J. (2012). Attitudes of teachers and headteachers towards inclusion in Lebanon. International Journal of Inclusive Education, 16(2), 139-153.

Kim, J. (2011). Influence of teacher preparation programmes on preservice teachers' attitudes toward inclusion. International Journal of Inclusive Education. 15(3), 355-377.

Kugelmass, J. (2004). The inclusive school: Sustaining equity and Standards. New York: Teachers College Press.

Kuyini, A., \& Desai, I. (2007). Principals' and teachers' attitudes and knowledge of inclusive education as predictors of effective teaching practices in Ghana. Journal of Research in Special Educational Needs, 7, 104-13.

Lempinen, J., \& Repo, J. (2002). Palestine (Gaza and West Bank) country report-education and training sector. Helsinki: Ministry for Foreign Affairs of Finland. Further Education Programme.

McDougall, J., D.J. DeWit, G. King, L.T. Miller, and S. Killip. (2004). High school-aged youths' attitudes towards their peers with disabilities: The role of school and student interpersonal factors. International Journal of Disability, Development and Education, 51(3), 287-313.

McMillan, J.H., \& Schumacher, S. (2001). Research in education: A conceptual introduction (5th Ed.). New York, NY: Longman.

Miles, S. (2005). Inclusive Education. Key issues and debates: Mainstreaming disability in development. The example of Inclusive Education. Phnom Penh: Cambodia.

Moran, A. (2007). Embracing inclusive teacher education. European Journal of Teacher Education, 30(2), 119-134.

Ojok, P., \& Wormnæs, S. (2012). Inclusion of pupils with intellectual disabilities: primary school teachers' attitudes and willingness in a rural area in Uganda. International Journal of Inclusive Education, (ahead of print), 1-19.

Palestinian Legislative Council (1999). Law on Rights of Disabled Palestinians. Avalilable from: http://dredf.org/international/PA-Disability-Law-Eng.pdf

Palestinian Ministry of Education (2008). Education Development Strategic Plan 2008-2012. Ministry of Education and Higher Education Palestine. Available from: http://planipolis.iiep.unesco.org/upload/Palestinian\%20Autonomous\%20Territories/Pa lestine_Education_development_plan_2008_2012.pdf 
Raphael, J., \& Allard, A.C. (2013). Positioning people with intellectual disabilities as the experts: enhancing pre-service teachers' competencies in teaching for diversity. International Journal of Inclusive Education, 17(2), 205-221.

Sentenac, M., Ehlinger, V., Michelsen, S., Marcelli, M., Dickinson, H., \& Arnaud ,C. (2013). Determinants of inclusive education of 8-12 year-old children with cerebral palsy in 9 European regions. Research in Developmental Disabilities, 34(1), 588-595.

Shevlin, M., Winter, E., \& Flynn, P. (2013). Developing inclusive practice: teacher perceptions of opportunities and constraints in the Republic of Ireland. International Journal of Inclusive Education, 17(10), 1119-1133.

Slee, R. (2011). The irregular school: Exclusion, schooling and inclusive education. Abingdon, UK: Routledge.

Thaver, T. \& Lim, L. (2012). Attitudes of pre-service mainstream teachers in Singapore towards people with disabilities and inclusive education. International Journal of Inclusive Education, ahead of print, 1-15. DOI:10.1080/13603116.2012.693399

UNESCO (1994). Final Report: World conference on special needs education: Access and quality. The Salamanca statement and framework for action on special needs education. París: UNESCO. UNESCO: Salamanca. Available from: http://unesdoc.unesco.org/images/0009/000984/098427eo.pdf.

UNESCO (2012a). The New Delhi Commitment: Delivering Inclusive, Relevant Quality Education for All. UNESCO: New Delhi. Available from: http://unesdoc.unesco.org/images/0021/002183/218359e.pdf

UNESCO (2012b). UNESCO National Education Support Strategy (UNESS). Palestine. UNESCO Ramallah Office and UNESCO Regional Bureau for Education in the Arab States.

United Nations (2006). Convention on the Rights of Persons with Disabilities. Available from: http://www.un.org/disabilities/convention/conventionfull.shtml

Vignes, C., Godeau, E., Sentenac, M., Coley, N., Navarro, F., Grandjean, H., et al. (2009). Determinants of students' attitudes towards peers with disabilities. Developmental Medicine and Child Neurology, 51(6), 473-479.

Villa, R.A. \& Thousand, J.S. (2005). Creating an Inclusive School. USA: Association for Supervision and Curriculum Development. 